Technical note

\title{
Divided-volume matching technique for volume displacement estimation of patient positioning in radiation therapy
}

\author{
Mutsumi Tashiro ${ }^{\mathrm{a}, \mathrm{b}, *}$, Yoshiki Kubota ${ }^{\mathrm{b}}$, Masami Torikoshi ${ }^{\mathrm{b}}$, Tatsuya Ohno ${ }^{\mathrm{b}}$, Takashi Nakano ${ }^{\mathrm{a}, \mathrm{b}}$ \\ ${ }^{\text {a }}$ Gunma University Initiative for Advanced Research, 3-39-22 Showa-Machi, Maebashi, Gunma 371-8511, Japan \\ b Gunma University Heavy Ion Medical Center, 3-39-22 Showa-Machi, Maebashi, Gunma 371-8511, Japan
}

\section{A R T I C L E I N F O}

\section{Keywords:}

Divided-volume matching

2D-3D image registration optimization

Patient positioning

Internal structure displacement

\begin{abstract}
A B S T R A C T
Purpose: We propose the Divided-Volume Matching (DVM) technique to visualize and estimate three-dimensional (3D) displacements of internal structures to enable more accurate patient positioning for radiation therapy.

Methods: A CT volume is divided into a volume of interest (VOI) and a base volume (BV); 2D-3D matching is achieved using digital radiography (DR) images and digitally reconstructed radiographs (DRRs), where the DRRs are iteratively generated by changing the 3D positions and rotation angles of the separate volumes independently to identify the best match with the DR images. We demonstrate this technique with two phantom and two clinical cases.

Results: 3D displacements of the VOIs could be estimated independently and simultaneously with those of the BVs, with accuracies comparable to those of the conventional 2D-3D matching. The proposed technique yielded more suitable matching results when internal displacements occurred in the regions of interest (ROIs). The best matches were found when the ROI was confined to the focused structure, initial displacement values were coarsely adjusted, one volume was matched while the other was fixed, or any combination thereof.

Conclusions: The proposed technique can be used effectively for independent displacement estimations of VOIs and BVs for patient positioning in radiation therapy.
\end{abstract}

\section{Introduction}

In radiation therapy, patient positioning is usually established by image registration that matches bony structures or fiducial markers between daily X-ray digital radiography (DR) images captured during treatments and digitally reconstructed radiographs (DRRs) derived from treatment planning (TP) computed tomography (CT) volume data. The displacement of parts of structures or organs in the CT data with respect to the base body structure inhibits matching of the overall structures. Such an outcome is particularly hazardous in particle beam radiotherapy, where structural changes or motions in the beam path may cause severe changes in the dose distribution in and around the target and organs at risk [1-5].

In actual treatment situations, however, perfectly matching a patient's anatomical structures with the CT data is quite difficult. For example, shoulder blades can be displaced after vertebral body matching is completed, or the diaphragm can be displaced even when DR images are captured at the same respiratory phase with gated irradiation. Displacements of more than $10 \mathrm{~mm}$ have been reported in lung anatomy at the same respiratory phase [1].

When large displacements in the internal structures are observed just before irradiation treatment, therapists must decide whether to proceed with the treatment, reposition the patient (if the displacement is found to be within the predetermined tolerance), or stop the treatment course and reschedule a new treatment according to the patient's present anatomical conditions. In such cases, quantifying the internal movement is required to make the most appropriate decision.

In recent years, two-dimensional-to-three-dimensional (2D-3D) matching techniques have been developed using orthogonal X-ray images and applied to patient positioning in radiotherapy clinics [6-9]. In the 2D-3D matching software, the displacement values can be automatically obtained by matching DRRs with DR images, where the DRRs are iteratively generated by changing the 3D positions and angles of the CT volume. Although the automatic registration is usually followed by manual adjustment and confirmation, it enables faster registration than only manual matching and operator-independent displacement estimation. However, in case that the internal structures, such as the target volume and surrounding organs displace with respect

\footnotetext{
* Corresponding author.

E-mail address: tashiro@gunma-u.ac.jp (M. Tashiro).
} 
to bony structures from the CT images, the matching optimization might be difficult to achieve. Especially, when both the target and the bony structures displace each other and overlap on the X-ray image, the matching optimization might be affected by both the structures, which might lead to matching failure. In such cases, operators have to adjust the matching regions of interest (ROIs) and/or manually match to obtain displacements of the bony structure and the target individually, when they need to estimate the target shift with respect to the bony structures.

In this paper, we propose a novel technique, called Divided-Volume Matching (DVM), to estimate the displacements of internal structures in addition to bony structures. The proposed technique was developed based on our 2D-3D patient positioning software [9] to visualize and quantify the shift of an internal structure by using CT and DR images. In the proposed technique, a CT volume is divided into two volumes: a volume of interest (VOI) and a base volume (BV). The VOI is supposed to involve an internal structure, e.g., an organ including the target volume, and the BV is composed of the remaining volumes, such as bony structures. Then, the 3D displacement of each volume is estimated by matching DRRs with DR images, where the DRRs are iteratively generated by changing the 3D positions and angles of the separated volumes independently. This technique is expected to be useful especially for particle beam radiotherapy, in which both matching of bony structures and quantifying the displacement of the internal structures are desired because they may influence the beam range and irradiated position, and consequently affect the dose delivery to the target and surrounding normal tissues. The DVM technique is validated through examinations of both phantom and clinical cases.

\section{Materials and methods}

\subsection{DVM technique}

In the proposed technique, a CT volume is divided into two volumes: a VOI and a BV. The basic principle of the technique is illustrated in Fig. 1. A VOI is established for the purpose of quantifying its independent motion and is segmented to include the structure that appears in different locations in the DR and TP-DRR images. The DR and DRR images are then processed to achieve 2D-3D matching. In this matching process, DRRs are iteratively generated by changing the $3 \mathrm{D}$ positions and rotation angles of both the VOI and BV independently to

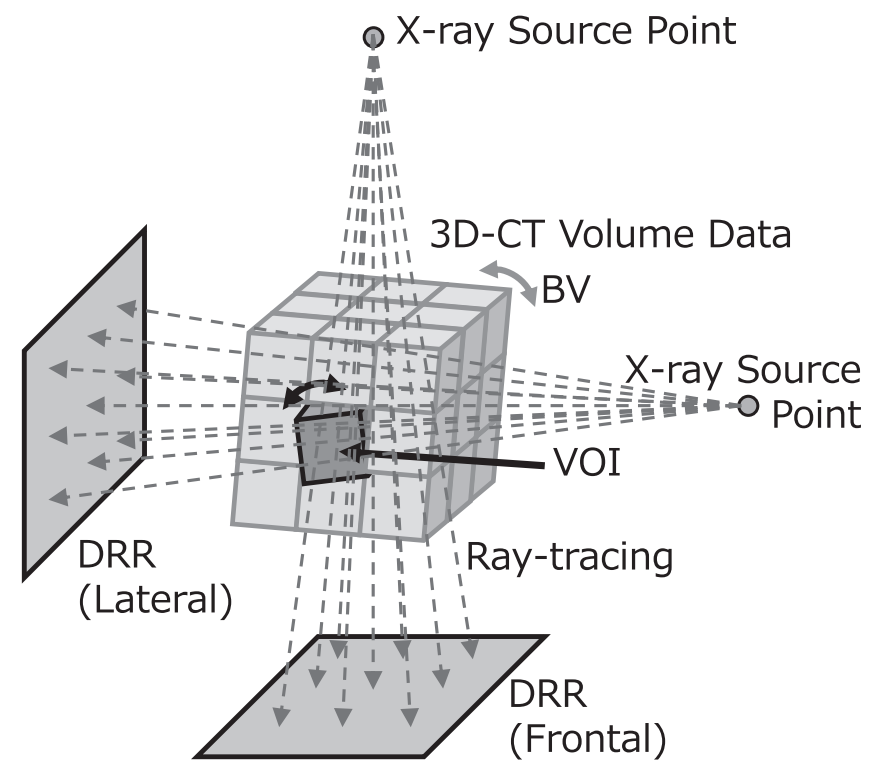

Fig. 1. DVM concept. (BV: base volume, VOI: volume of interest, DRR: digitally reconstructed radiograph, 3D-CT: three dimensional computed tomography). identify the best match with the DR images, deriving 12 motion parameters (three translations and three rotation angles for two volumes).

To generate the DRRs, a CT volume, in which the BV and the VOI are translated and rotated independently, is derived. The CT values after displacements of the BV and the VOI are expressed as

$C T\left(\boldsymbol{r} \in \mathrm{BV}^{\prime}\right)=B V^{\prime}(\boldsymbol{r})=C T_{0}\left(\boldsymbol{r}^{\prime}\right)$,

$C T\left(\boldsymbol{r} \in \mathrm{VOI}^{\prime \prime}\right)=\operatorname{VOI}^{\prime \prime}(\boldsymbol{r})=C T_{0}(\boldsymbol{r})$,

where $r$ is the position in the CT volume; $B V^{\prime}$ and VOI" are the CT values of the BV and the VOI, respectively, displaced from the CT volume at the original position, $C T_{0}$; and $\boldsymbol{r}^{\prime}$ and $\boldsymbol{r}^{\prime \prime}$ are the original positions before the respective displacements, expressed as follows.

$\boldsymbol{r}=H_{\mathrm{BV}} \boldsymbol{r}^{\prime}$ (forBV)

$\boldsymbol{r}=H_{\mathrm{BV}} H_{\mathrm{VOI}} \boldsymbol{r}^{\prime \prime}($ forVOI)

Here, $H_{\mathrm{BV}}$ and $H_{\mathrm{VOI}}$ are $4 \times 4$ matrix transformations that represent $3 \mathrm{D}$ displacements comprising both the translation and the rotation of the BV and VOI, respectively; the position vector $r$ can be expressed in matrix form as

$\boldsymbol{r}=\left(\begin{array}{llll}x & y & z & 1\end{array}\right)^{T}$,

where $T$ denotes the transposed matrix. As shown in Eq. (4), the transformation of the VOI is executed sequentially, so that the displacement amounts of the VOI are given with respect to the BV position; if all the displacement amounts of the VOI are zero, the VOI is displaced together with the $\mathrm{BV}$ as one rigid volume. The transformation matrix $H$ ( $H_{\mathrm{BV}}$ and $H_{\mathrm{VOI}}$ ) is given as

$$
\begin{aligned}
H= & H_{T}\left(x_{0}, y_{0}, z_{0}\right) H_{t}(\Delta x, \Delta y, \Delta z) H_{x}\left(\Delta \theta_{x}\right) H_{z}\left(\Delta \theta_{z}\right) H_{y}\left(\Delta \theta_{y}\right) H_{T}^{-1} \\
& \left(x_{0}, y_{0}, z_{0}\right),
\end{aligned}
$$

where $H_{\mathrm{x}}, H_{\mathrm{y}}$, and $H_{\mathrm{z}}$ are the rotation matrices around the $x$-, $y$-, and $z$ axes, respectively, indicating pitch, roll, and rotation; $H_{\mathrm{t}}$ is the translation matrix; and $H_{\mathrm{T}}$ is the translation matrix that sets the center of rotation $\left(x_{0}, y_{0}, z_{0}\right)$. The rotation centers of the $\mathrm{BV}$ and VOI are set as the iso-center and the center of the VOI, respectively.

When the BV and the VOI are displaced independently, overlapping and empty volumes can be formed between them. To prevent the loss of structures with higher densities, such as bony structures, the diaphragm (in lung images), radio-contrast agents, and radio-opaque fiducial markers, all of which can be guideposts for image registration, the CT values at overlapping volumes are set as the higher values in the two volumes as follows.

$C T(\boldsymbol{r} \in$ overlap $)=\max \left[B V^{\prime}(\boldsymbol{r}), V^{\prime \prime \prime}(\boldsymbol{r})\right]$.

To prevent higher density structures from remaining at their original positions in empty volumes as well as their displaced positions, and appearance of unreasonable voids in DRRs, the CT values at empty positions are filled with the lower CT values as follows.

$C T(\boldsymbol{r} \in$ void $)=\min \left[B V^{\prime}(\boldsymbol{r}), \operatorname{VOI}^{\prime \prime}(\boldsymbol{r})\right]$.

In the ray-tracing process for DRR generation, the VOI is displaced with respect to the fixed $\mathrm{BV}$ position, and the ray positions are also inversely transformed according to the $\mathrm{BV}$ transformation in order to reduce the computation cost. The CT values at the ray positions are obtained by tri-linear interpolation, and the pixel values of the DRRs are obtained by accumulating the CT values along the respective rays in 1-mm steps, rescaling all accumulated pixel values to 8-bit resolutions.

In the DVM process, the 3D displacement amounts (including both translations and rotation angles) of the BV and the VOI are derived by image matching between the DRRs and DR images of the frontal and lateral directions. The matching optimization is done to minimize an objective function,

$f=1-\left(Z N C C_{F}+Z N C C_{L}\right) / 2$, 

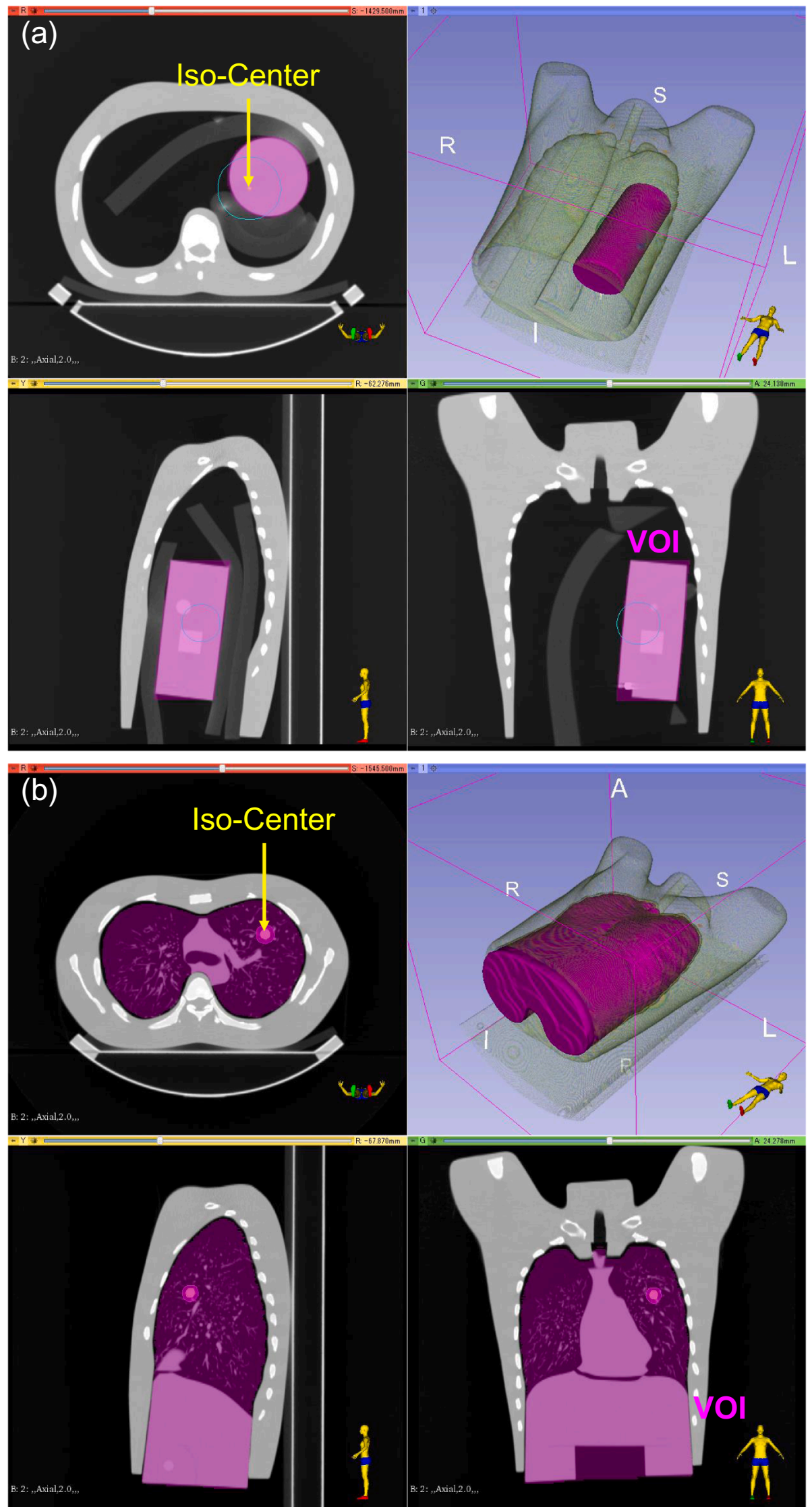

Fig. 2. CT images of axial (upper left), sagittal (lower left), and coronal (lower right) planes for Case 1: chest phantom with imaging insert (a), Case 2: chest phantom with mediastinum and lung (b), Case 3: head and neck cancer patient (c), and Case 4: liver cancer patient (d). The purple volumes indicate the VOIs. The upper right images of respective cases are stereoscopic displays to show the positional relationships. (VOI: volume of interest). (For interpretation of the references to colour in this figure legend, the reader is referred to the web version of this article.) 

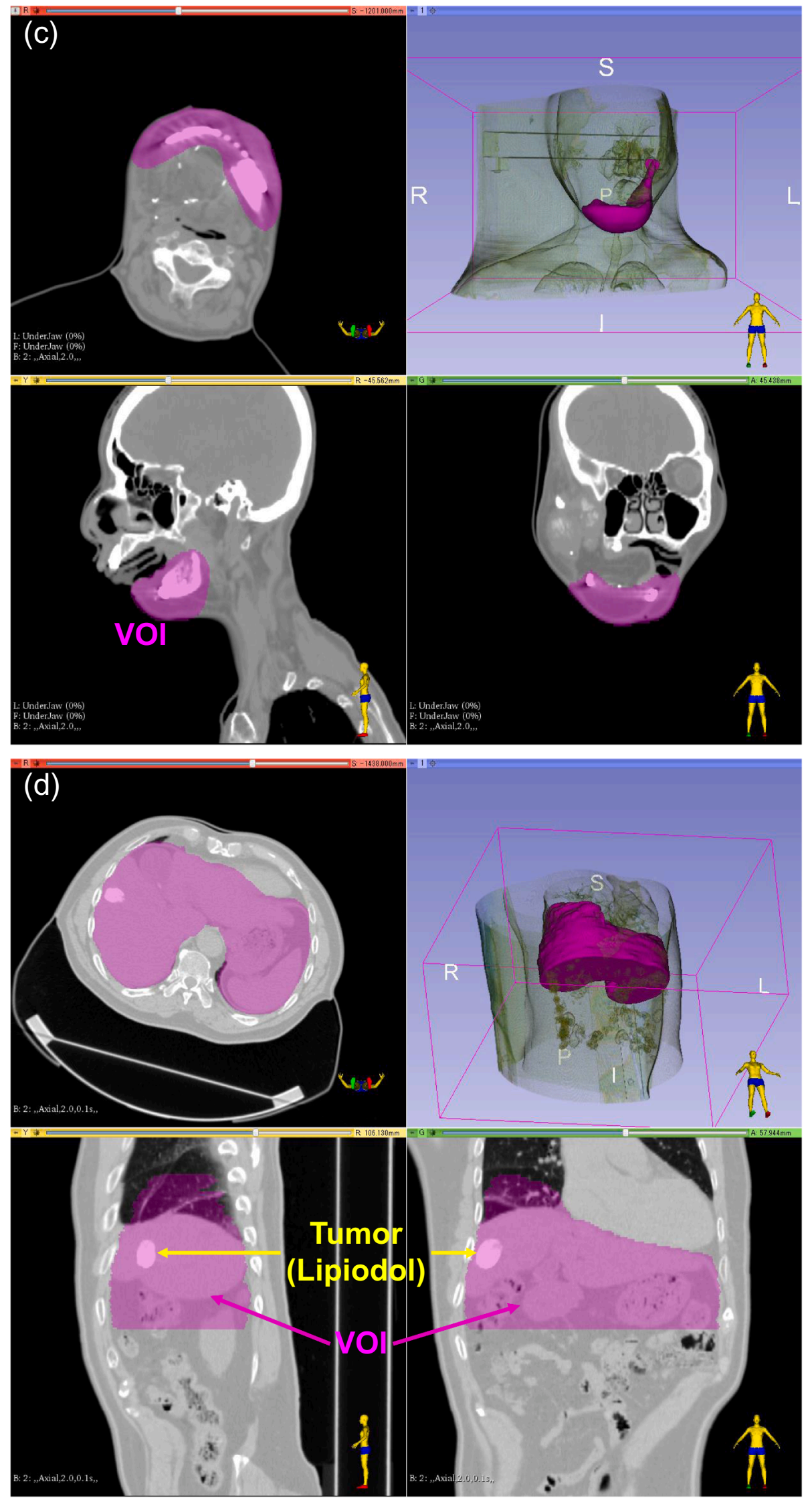

Fig. 2. (continued) 
where $Z N C C_{F}$ and $Z N C C_{L}$ are zero-means normalized cross-correlations between the two images for the frontal and lateral directions, respectively, and are calculated from the pixel values within the regions of interest (ROIs) set on the respective images. The steepest descent and golden section methods are used for the matching optimization.

\subsection{Examination of DVM}

We demonstrated the proposed technique with two phantom and two patient cases. For all cases, the CT images and VOIs are shown in Fig. 2(a)-(d) to display the positional relationships of the VOIs in the CT volumes. This study was approved by the institutional review board (approval number HS2018-135) of Gunma University Hospital.

\subsubsection{Case 1: Chest phantom with imaging insert}

A chest phantom N-1 (Kyoto Kagaku Co., Ltd, Kyoto, Japan) and the four-dimensional (4D) CT imaging insert for the QUASAR ${ }^{\mathrm{TM}}$ phantom (Modus Medical Devices, Inc., London, Canada) were used as the BV and the VOI, respectively. The imaging insert was inserted into the left side of the chest phantom. X-ray CT scan was performed and a simplified treatment plan was established to assign an iso-center position in the CT volume for DRR generation. The phantom on the couch was then shifted to the treatment position for patient positioning. To introduce a VOI displacement, the imaging insert was manually shifted in the chest phantom and orthogonal X-ray images were captured for DVM examination. The volume of the imaging insert was segmented as a VOI in the CT volume.

\subsubsection{Case 2: Chest phantom with mediastinum and lung}

The chest phantom and an accompanying assembly of mediastinum and lung were used as the BV and the VOI, respectively. The procedure was basically the same as that used for Case 1 . The VOI displacement was introduced by manually sliding the assembly in the inferior direction. The mediastinum and lung volume were segmented as the VOI.

\subsubsection{Case 3: Head and neck cancer patient}

A data set containing CT volume data, treatment plan data, and DR images from a treatment course was obtained for a head and neck cancer patient, who had a tumor in the right malar region and received carbon-ion beam therapy $[10,11]$. In this case, displacement of the mandible adjacent to the tumor was seen to be a problem during patient positioning for irradiation treatment. The mandible and surrounding tissues were segmented as the VOI in the CT volume. A set of orthogonal X-ray images captured during patient positioning for irradiation treatment was used for DVM examination.

\subsubsection{Case 4: Liver cancer patient}

A data set containing CT volume data, treatment plan data, and DR images from a treatment course was obtained for a liver cancer patient treated by carbon-ion beam therapy. In this case, the treatment problem was inter-fractional reproducibility of the liver position at the exhale peak phase, even though respiratory gating was applied. The tumor could be visualized in X-ray images by using the contrast agent Lipiodol. The lower parts of the lungs and the inferior side of the liver, which appeared to move together, were segmented as the VOI in the CT volume. As in Case 3, a set of orthogonal X-ray images captured on the day of treatment was used for DVM examination.

\subsubsection{Examination and error evaluation}

In all cases, DVM examinations were conducted under the following optimization conditions: only the whole CT volume (VOI + BV) matching (i.e., conventional 2D-3D matching), simultaneous $\mathrm{BV}$ and VOI matching, and only VOI matching after BV manual matching. Other specific conditions were applied for the respective cases, as summarized in Table 1. For comparison, the conventional 2D-3D matching was conducted using the same matching ROI by fixing the six displacement values of the VOI to zero. The matching ROIs were set in basically rectangular shapes as widely as possible within the collimators on the DR images, avoiding regions with saturated pixel values, except for the specified matching conditions described in the text. The window width and window level settings of the DRRs were fixed for all matching conditions in each case, after they were adjusted to match approximately with the contrast in the DR images. For the matching process, the sizes of DR images and DRRs were set to $256 \times 256$ pixels, corresponding to $0.89 \mathrm{~mm} / \mathrm{pixel}$ at the iso-center plane. The computations for matching optimization were performed using a general workstation with dual CPUs (Intel Xeon E5-2670 2.6 GHz), $32 \mathrm{~GB}$ memory, and a GPU (NVIDIA Tesla M2090 (512 CUDA cores, 6 GB memory)).

In order to evaluate the matching accuracies, reference displacement data were obtained from manual matching by radiation technologists. Because the displacement values of the VOIs obtained from Eq. (4) represented those with respect to the relevant BVs, the absolute displacement values with respect to the original position were obtained using the following conversion formula:

$H_{\mathrm{VOI}}^{\text {absolute }}=H_{\mathrm{BV}} H_{\mathrm{VOI}}$,

where the rotation center of the left-hand side was set to the origin (isocenter). The errors of the resultant displacements were then estimated with respect to the reference data (three translation errors $(\Delta \mathrm{X}, \Delta \mathrm{Y}, \Delta \mathrm{Z})$ and three rotation angle errors $\left.\left(\Delta \theta_{X}, \Delta \theta_{Y}, \Delta \theta_{Z}\right)\right)$.

\section{Results}

Figs. 3-6 show (a) the initial DRRs generated from the CTs, (b) the generated DRRs after simultaneous DVM as examples of the matching results, and (c) DR images at patient positioning for Cases 1-4, respectively. The errors of the displacements relative to the reference data and root mean square errors (RMSEs) of the translations and rotations for the BVs and VOIs are summarized in Table 1. In the condition column, "Simul. DVM" indicates simultaneous VOI and BV matching, and "fixed BV" means that the BV position was fixed to the reference position and only the VOI matching optimization was performed. The computation times of matching optimizations for the respective conditions are summarized in Table 1 . The computation time was determined from the number of iterations for optimization convergence and the machine specification, which might be correlated with the iterative generation of DRRs.

\subsection{Chest phantom with imaging insert (Case 1)}

The RMSEs for simultaneous DVM (VOI and BV) (Condition 1b in Table 1) were smaller than those obtained using the conventional matching approach with one CT volume (Condition 1a). The proposed approach produced RMSEs of less than $1 \mathrm{~mm}$ and $1^{\circ}$, except for the VOI rotation. The errors did not change as much as those for VOI matching with fixed BV (Condition 1c). These results were attributed to the comparatively large errors of $\Delta \theta y$ for the VOI with a cylindrical shape, which inhibited the correct convergence for the cylindrical rotation. By adjusting the initial angle of $\theta y$ to $-5^{\circ}$, nearer to the reference value, the results improved significantly for both simultaneous DVM (Condition 1e) and VOI matching with fixed BV (Condition 1d).

\subsection{Chest phantom with mediastinum and lung (Case 2)}

In this case, the RMSEs for simultaneous DVM (Condition 2b) were also smaller than those obtained using conventional matching (Condition 2a): less than $1 \mathrm{~mm}$ and $1^{\circ}$ on average. In particular, the positional accuracy of the VOI improved for simultaneous DVM $\left(3.16 \mathrm{~mm}\right.$ and $0.94^{\circ}$ to $0.66 \mathrm{~mm}$ and $\left.0.49^{\circ}\right)$. The errors further decreased to $0.24 \mathrm{~mm}$ and $0.18^{\circ}$ for VOI matching with fixed BV (Condition 2c). 


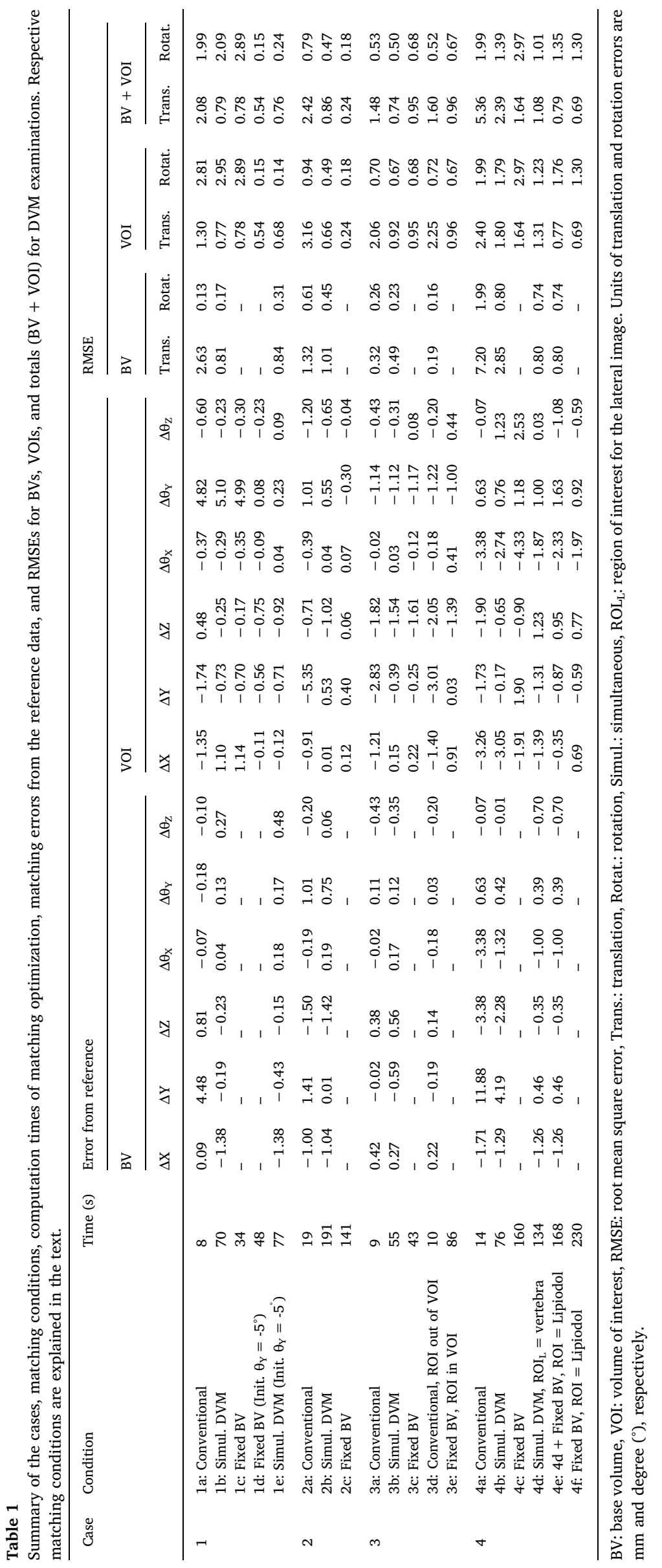



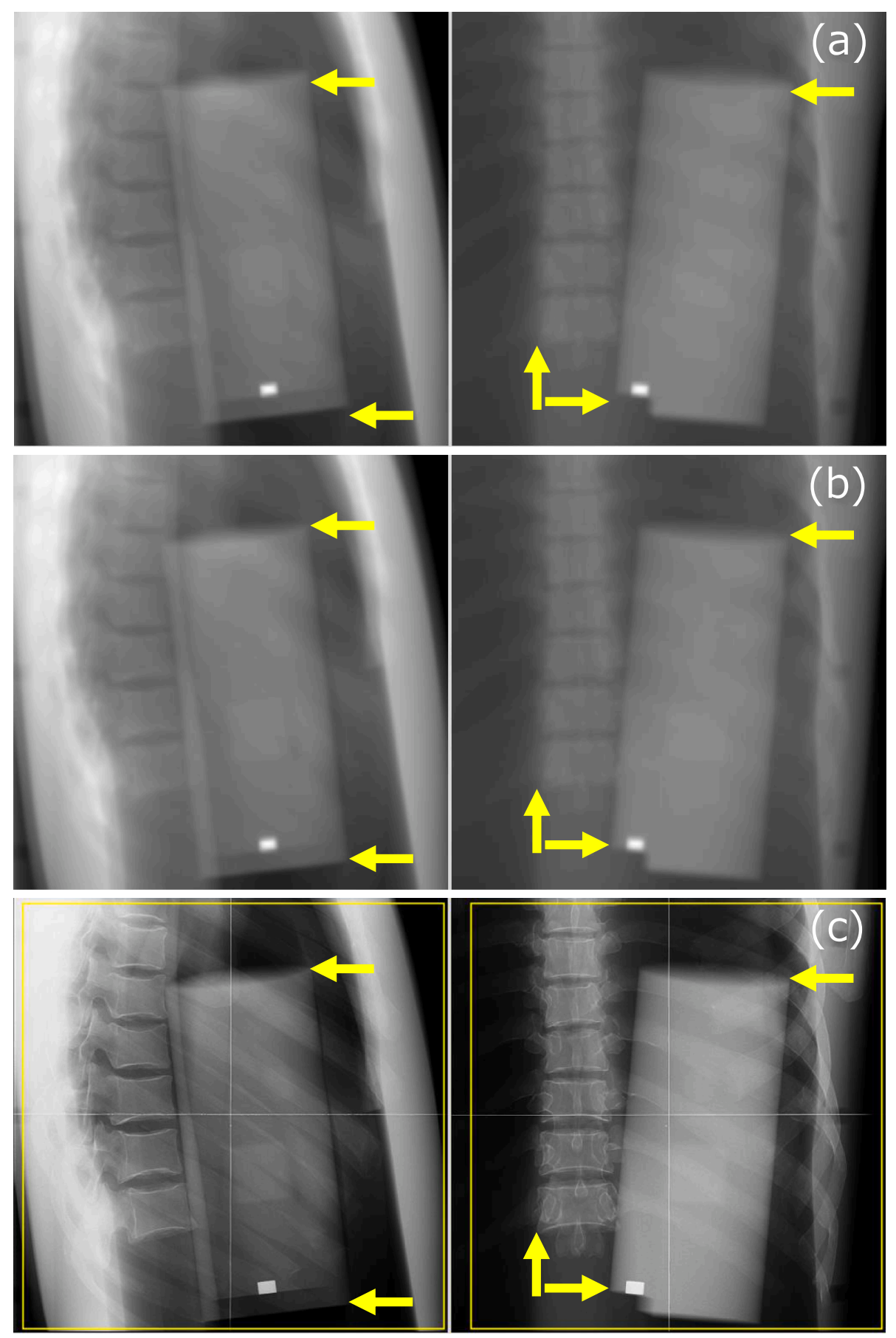

Fig. 3. (a) Initial DRRs generated from CTs, (b) DRRs after simultaneous DVM, and (c) DR images at patient positioning for Case 1: chest phantom with imaging insert. Left and right sides show lateral and frontal images, respectively. The matching ROIs are indicated by the yellow boxes in the DR images (c). Yellow arrows are drawn in the same positions to highlight structural displacements among the images. (For interpretation of the references to colour in this figure legend, the reader is referred to the web version of this article.)

\subsection{Head and neck cancer patient (Case 3)}

As in the phantom cases, the RMSEs for simultaneous DVM (Condition $3 \mathrm{~b}$ ) were smaller than those obtained using conventional matching (Condition 3a). The accuracy of the mandible VOI improved in the simultaneous DVM. Even for VOI matching with fixed BV (Condition 3c), the RMSEs of the VOI were similar to those for the simultaneous DVM: less than $1 \mathrm{~mm}$ and $0.7^{\circ}$.

When the matching ROI was confined to outside the mandible, the RMSEs of the BV obtained using the conventional one-volume matching approach further decreased to less than $0.2 \mathrm{~mm}$ and $0.2^{\circ}$ (Condition 3d). However, when the ROI was confined to the mandible region (Condition 3e), VOI matching with fixed BV showed RMSEs of the VOI as $0.96 \mathrm{~mm}$ and $0.67^{\circ}$, which were similar to those for simultaneous DVM.

\subsection{Liver cancer patient (Case 4)}

In manual VOI matching for reference data acquisition, simultaneously matching all structures in the VOI, including the diaphragm, radio-contrast agent Lipiodol, and radio-opaque fiducial marker, was not feasible because of the intrinsic liver deformation from the CT. 

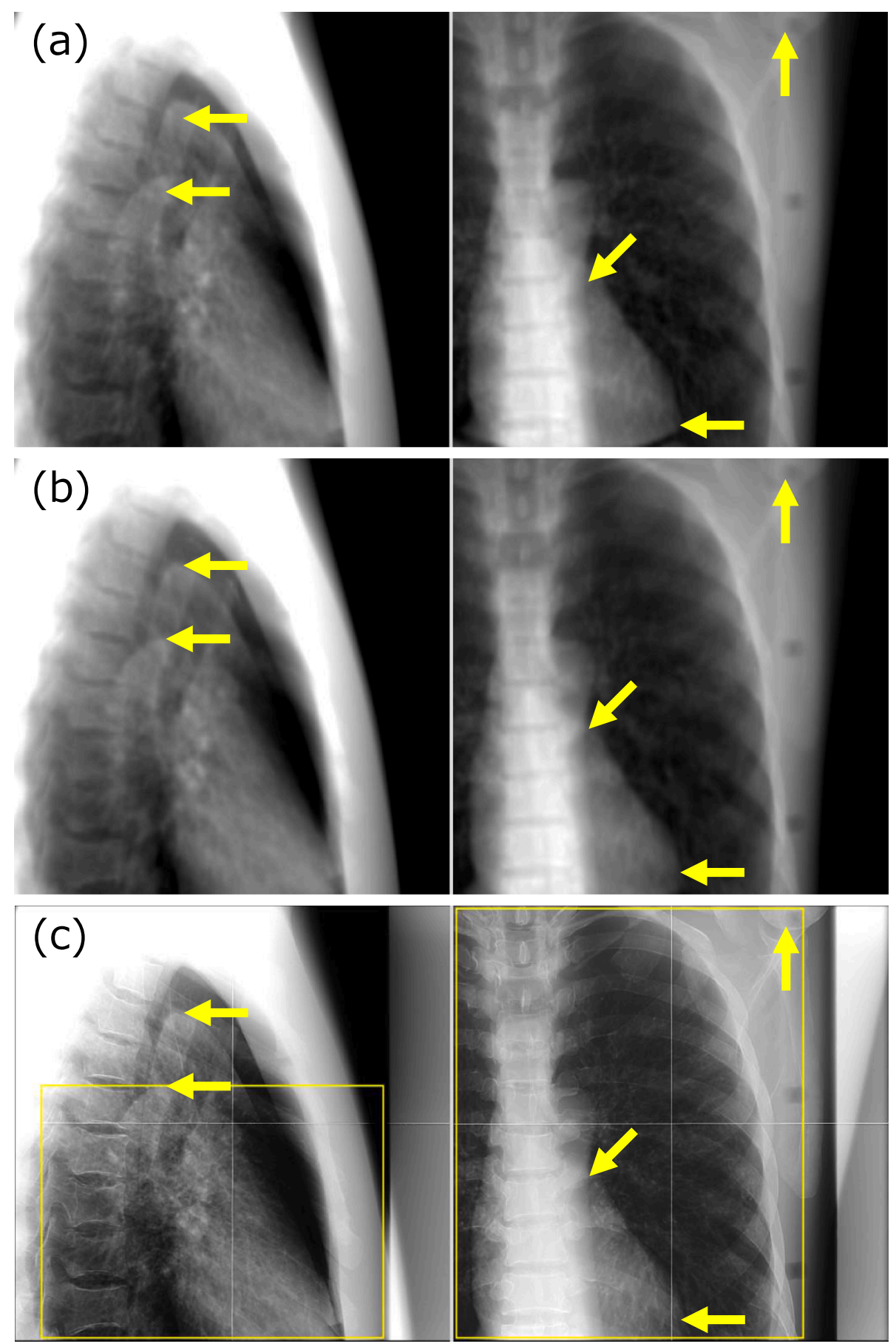

Fig. 4. (a) Initial DRRs generated from CTs, (b) DRRs after simultaneous DVM, and (c) DR images at patient positioning for Case 2: chest phantom with mediastinum and lung. Left and right sides show lateral and frontal images, respectively. The matching ROIs are indicated by the yellow boxes in the DR images (c). Yellow arrows are drawn in the same positions to highlight structural displacements among the images. (For interpretation of the references to colour in this figure legend, the reader is referred to the web version of this article.)

Therefore, in this study, we focused on Lipiodol, which indicated the tumor position, and adopted the results of manual matching with Lipiodol as the reference.

The RMSEs for Case 4 were comparatively larger than those for the other cases; however, like the other cases, the RMSEs for simultaneous DVM (Condition 4b) were smaller (translation: $2.39 \mathrm{~mm}$, rotation: $1.39^{\circ}$ ) than those obtained using the conventional matching (Condition 4a). For VOI matching with fixed BV (Condition 4c), the accuracy of VOI matching did not improve compared to that for Condition $4 \mathrm{~b}$. Because the VOI was segmented largely in the abdomen, deformation in the liver and surrounding tissues actually occurred in the matching region. Therefore, the matching may have been affected by such nonrigid displacements, especially the displacements of the diaphragm and Lipiodol, and changes in the image contrast with the surrounding tissues. Setting a wide matching ROI to include such deformation would limit the matching accuracy of the VOI with deformation.

To determine the improvement of accuracy suggested from the above results, the matching ROI for the lateral image was confined to the vertebral body and simultaneous DVM was examined (Condition 4d) to match the BV first. In this condition, the translation RMSE of the 

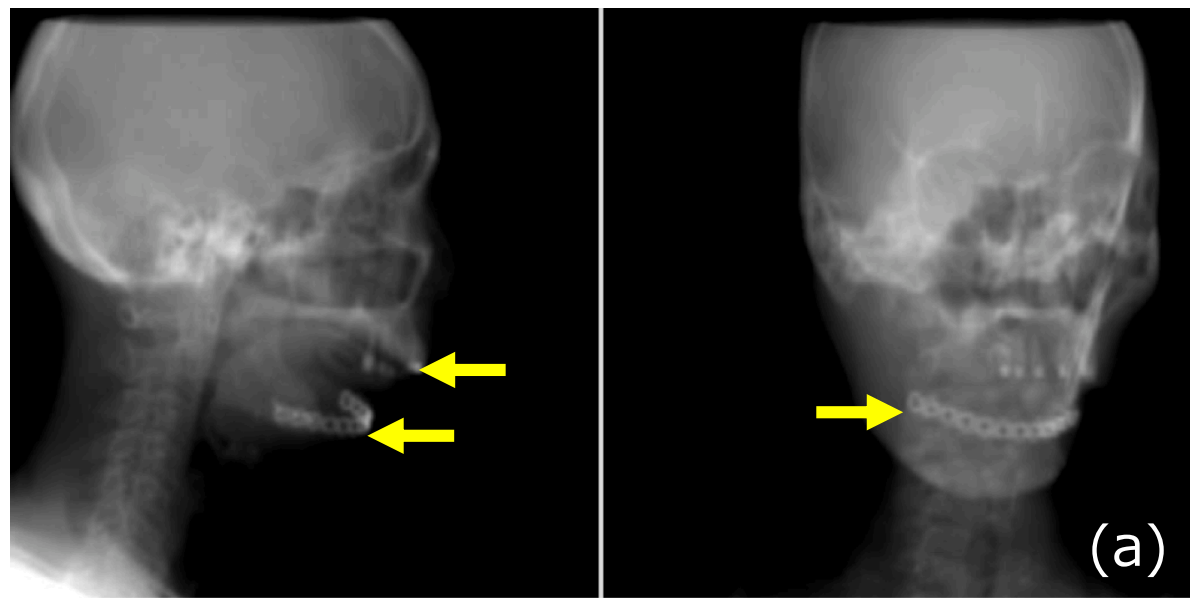

Fig. 5. (a) Initial DRRs generated from CTs, (b) DRRs after simultaneous DVM, and (c) DR images at patient positioning for Case 3: head and neck cancer patient. Left and right sides show lateral and frontal images, respectively. The initial matching ROIs, the ROIs confined to avoid the mandible, and the ROIs confined to the mandible are indicated by the yellow, dashed green, and red boxes in the DR images (c), respectively. Yellow arrows are drawn in the same positions to highlight structural displacements among the images. (For interpretation of the references to colour in this figure legend, the reader is referred to the web version of this article.)
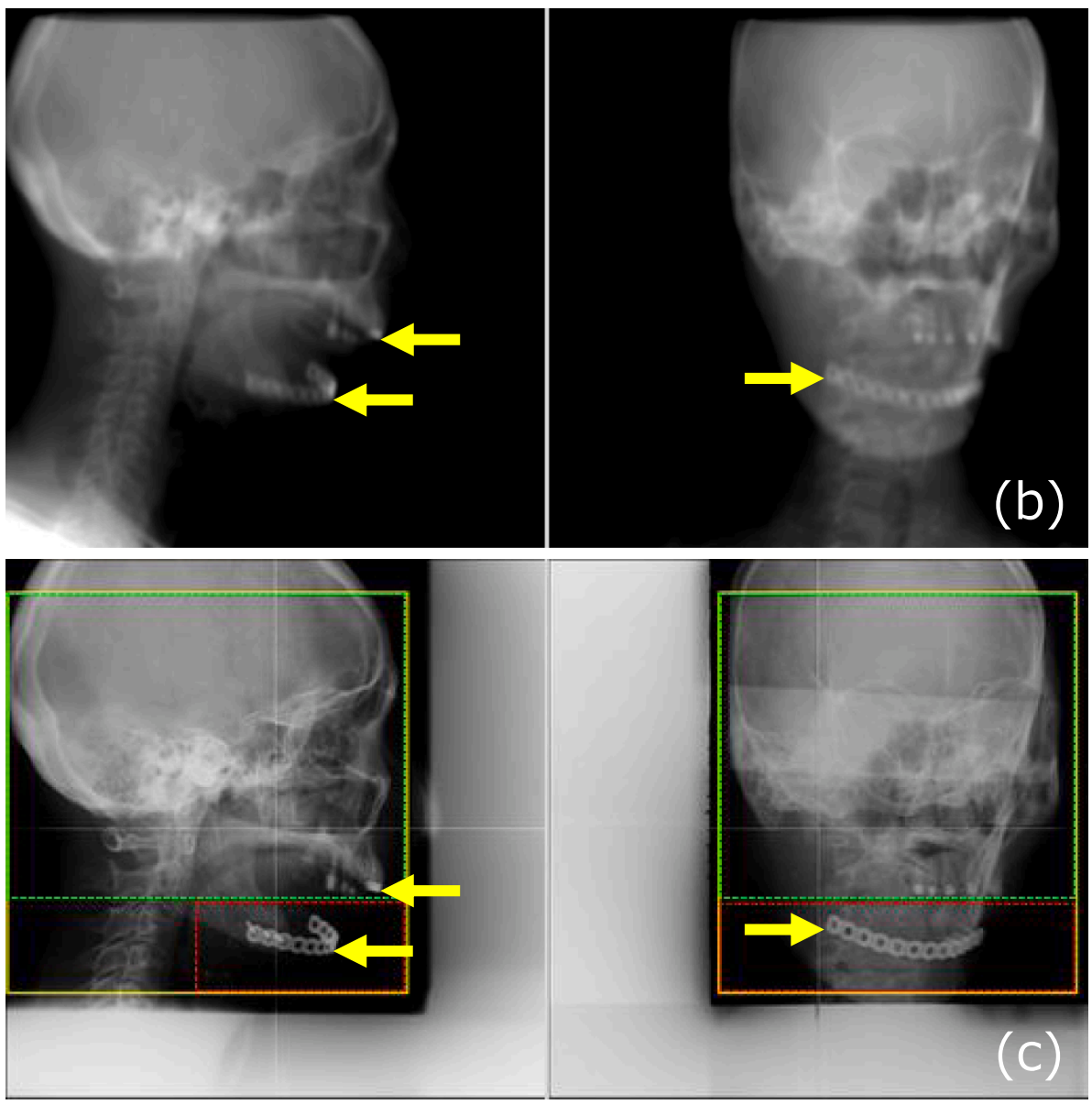

BV significantly reduced from $2.85 \mathrm{~mm}$ to $0.80 \mathrm{~mm}$. Subsequently, the ROIs of both the frontal and lateral directions were confined to Lipiodol and a small surrounding area, and VOI matching was examined (Condition 4e); the RMSE of the VOI was reduced for translation, from $1.80 \mathrm{~mm}$ to $0.77 \mathrm{~mm}$, although the rotation RMSE did not change considerably $\left(1.79^{\circ}\right.$ to $\left.1.76^{\circ}\right)$. VOI matching with BV fixed to the reference position using the same confined ROIs (Condition $4 \mathrm{f}$ ) slightly reduced the RMSEs to $0.69 \mathrm{~mm}$ and $1.30^{\circ}$. The slightly larger RMSE of rotation was attributed to insufficient image information for correct convergence caused by confining the ROIs to the Lipiodol with the spherical tumor shape.

\section{Discussion}

Simultaneous DVM produced more reasonable and correct results compared with the conventional 2D-3D matching approach in all cases. Therefore, the DVM technique offers advantages over the conventional one not only for internal displacement estimation but also for practical patient positioning in cases where internal displacements are involved in matching ROIs. However, the DVM requires optimizing 12 displacement value parameters for two volumes, whereas the conventional approach uses only six parameters. Therefore, the convergence to the appropriate displacement values for DVM would be more difficult because the increased number of parameters increases the possibility of convergence to a certain local minimum, the number of which can also be expected to increase. This is suggested in our results, which indicate 

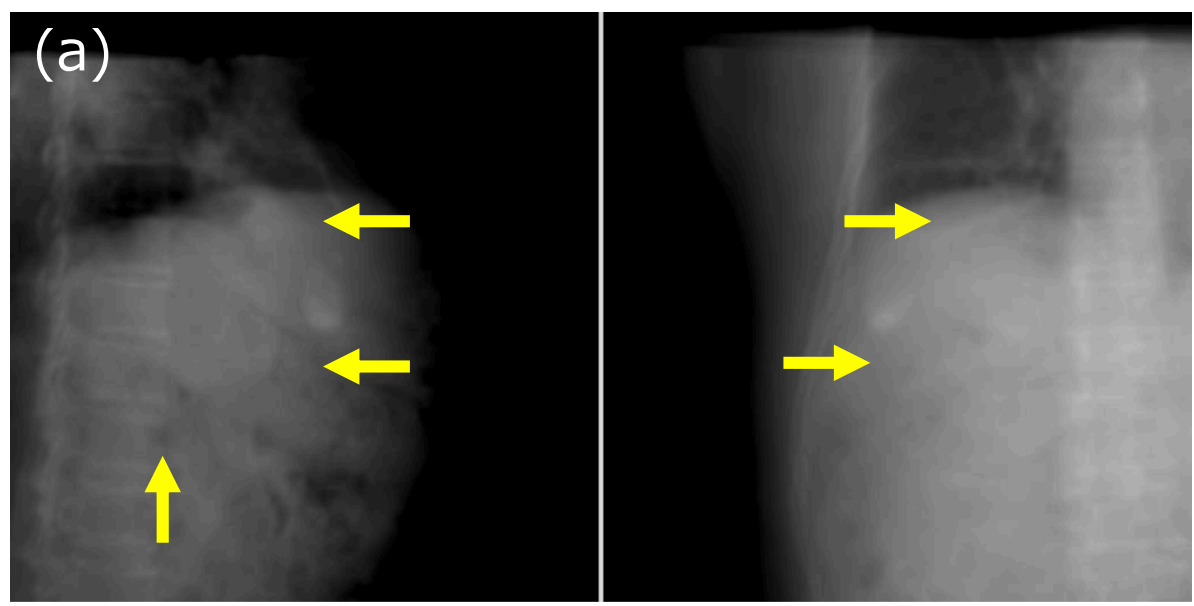

Fig. 6. (a) Initial DRRs generated from CTs, (b) DRRs after simultaneous DVM, and (c) DR images at patient positioning for Case 4: liver cancer patient. Left and right sides show lateral and frontal images, respectively. The initial matching ROIs, the ROI confined to vertebra for the lateral image, and the ROIs confined to the Lipiodol are indicated by the yellow, dashed green, and red boxes in the DR images (c), respectively. Yellow arrows are drawn in the same positions to highlight structural displacements among the images. (For interpretation of the references to colour in this figure legend, the reader is referred to the web version of this article.)
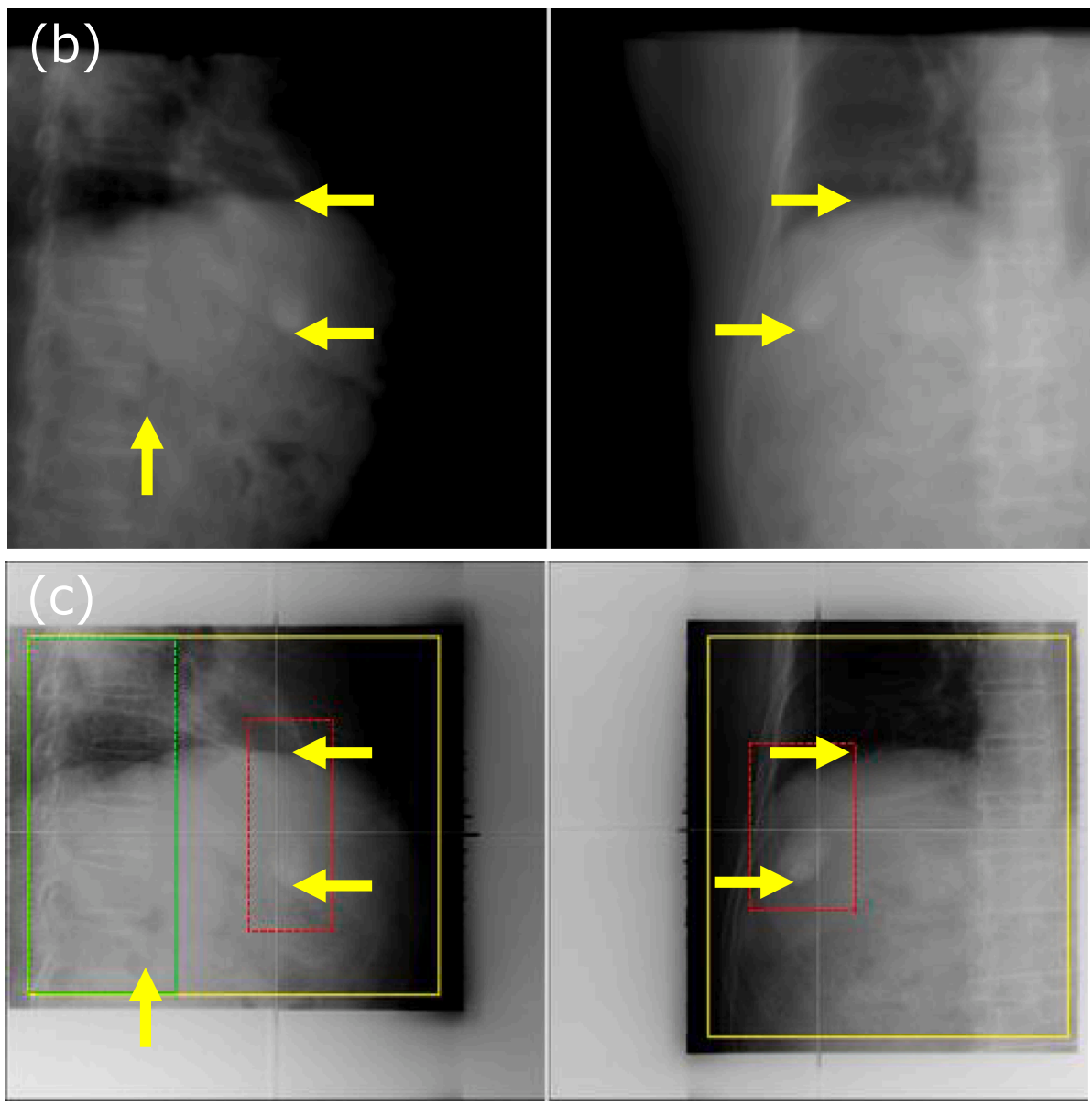

that the VOI accuracies are improved by setting the initial rolling angle near the appropriate value as shown in Case 1, or by fixing the BVs as shown in Cases 1 and 2. Adjustment and confinement of matching ROIs enables us to evaluate displacements of the focused volumes more appropriately, as shown in Cases 3 and 4. In addition to ROI adjustments, the combined use of manual setting of the initial displacements to appropriate values and/or matching with one fixed volume parameters might also facilitate appropriate convergence, improving the accuracy of displacement evaluation, which may be useful for practical applications. In practice, the large error convergence would be suppressed by initial patient setting to laser markers in the irradiation rooms and by roughly adjusting the initial displacement values of the volumes with confirmed DRRs and DR images.

In practical situations of patient positioning, various approaches can be attempted for image registration. With adjusting the ROI size and position, it might be also possible to match the image with bony structure and the target individually by the conventional matching technique. However, in case that the target (VOI) and bony structures, such as rib or spine (BV) are displaced from the treatment planning CT and overlapped on the X-ray image, mismatching of the image is unavoidable even when the matching ROI is confined to the target region. Even using such registration steps, both VOI and BV cannot be matched simultaneously and the total RMSE cannot be minimized with the conventional registration technique. In contrast, the developed technique showed more reasonable matching results for both VOI and BV simultaneously, even when the large matching ROIs were broadly set. This shows the potential of the proposed technique for both the purposes of matching bony structures and quantifying the displacement of 
the internal structure.

The accuracies of the conventional 2D-3D matching approach have been reported for various clinical cases. Kubota et al. reported the following worst case RMSEs for translation and rotation: approximately $0.2 \mathrm{~mm}$ and $0.2^{\circ}$ for a head phantom, and for clinical cases, $0.5 \mathrm{~mm}$ and $0.4^{\circ}$ for head and neck, $0.5 \mathrm{~mm}$ and $0.5^{\circ}$ for prostate, $1.4 \mathrm{~mm}$ and $0.8^{\circ}$ for lung, $3.4 \mathrm{~mm}$ and $2.0^{\circ}$ for liver, and $6.0 \mathrm{~mm}$ and $3.5^{\circ}$ for pancreas cancer patients [9]. In the phantom cases in our results, the RMSEs for simultaneous DVM were derived as $0.5-1 \mathrm{~mm}$ for translation and $0.2-0.5^{\circ}$ for rotation, except for the rolling cylinder in Case 1 . The large error of the cylinder rolling angle was also improved by setting the initial angle to approximate the appropriate value. These results were shown to be comparable with those of conventional matching. In the clinical case of head and neck cancer patient, RMSEs for the VOI were $0.96 \mathrm{~mm}$ and $0.67^{\circ}$, even with the ROI confined to the mandible and the fixed BV condition, and not substantially changed from the values for the simultaneous DVM and the fixed BV with the initial large ROIs. This may be influenced by the structural shifts observed by comparing the DRRs after simultaneous DVM, in Fig. 5(b), and the DR images at the patient position, in Fig. 5(c). These shifts may have occurred in the cervical vertebrae but could not be definitively located, although they occurred even in the confined ROI for the frontal images. In the liver cancer patient case, the comparatively large errors might be influenced by the deformations of the liver and surrounding anatomies that appeared in the DR images. Nevertheless, in the clinical cases, the total RMSEs for simultaneous DVM were derived as $0.7 \mathrm{~mm}$ and $0.5^{\circ}$ for the head and neck case and $2.4 \mathrm{~mm}$ and $1.4^{\circ}$ for the liver case, which were comparable with the reported values obtained using the conventional method. These results support the applicability and efficacy of the proposed DVM technique.

In recent years, cone-beam (CB) CT has been widely used for target positioning in image-guided radiotherapy, and some clinics are equipped with in-room $\mathrm{CT}$, including particle beam radiotherapy facilities [12-14]. Time and imaging strategies for target positioning are reported for sophisticated radiotherapy procedures including СВCT and mega-voltage CT functions $[15,16]$. In addition to rigid registration for patient positioning, the CT-CT deformable image registration (DIR) function, which leverages a pair of 3D-CT volume data, has also been successfully used to visualize and quantify three-dimensional (3D) internal structure motion. This might be also advantageous for dose distribution monitoring at treatment irradiation [17]. Such CT functions and applications might be powerful tools for displacement estimation of internal structures. However, such applications require an additional data set of CT images, and such CT functions in irradiation rooms are largely unavailable especially in conventional particle beam radiotherapy facilities $[1,10,18,19]$. Instead, such facilities are mostly equipped with two orthogonal X-ray imaging systems and 2D DR images can be obtained in a few seconds. Moreover, CT scanning may cause additional radiation exposure to patients $[20,21]$. In such situations, the proposed technique might have a potential for both registration of bony structures and displacement estimation of an internal structure, without additional CT scanning.

Some limitations of the proposed technique were observed in the present study. First, only two volumes can be accepted for the simultaneous displacement evaluation, and only rigid displacements of the volumes are possible. Increasing the number of volumes and/or implementing non-rigid deformation of CT volumes may further challenge internal motion estimations in larger volumes. In practice, deformable registration between a CT volume and the rigidly deformed volume after DVM could be useful for obtaining more appropriate CT volumes by eliminating discontinuous interfaces among the separated volumes, although the processes adopted here to address overlapping and empty volumes functioned adequately for obtaining 2D images without undue influence from the interfaces, as shown in the generated DRRs. The scope for application of the connection process among the separated volumes should be considered in further investigations for appropriate image registration and in practical use. Although the number of volumes and the manner of volume displacement are limited in the present technique, the DVM approach is shown to improve positioning accuracy of the BVs and the displacement evaluations of VOIs in clinical cases involving internal displacements.

A second potential limitation is that the VOIs for displacement evaluations have to be segmented before DVM execution. Segmentation would be a time-consuming procedure compared with the other methods, such as CT-CT DIR for the purpose of estimating the displacement of the internal structure. To address this problem, contouring performed usually in TP, in conjunction with additional advances of volumes that are expected to move, might be useful. Furthermore, implementing graphical user interface functions to assign and edit the volumes into the DVM software might be more useful in practice.

Third, the displacement evaluation is limited to the VOI and BV structures that can be observed on X-ray images. This is a general and intrinsic problem for X-ray image registration. For example, direct matching of lung tumor images might be quite difficult; however, matching of diaphragm or metal markers would be possible. Recently, markerless tumor tracking techniques using artificial intelligence and other techniques that enable estimation of almost invisible tumor shapes and positions in 2D-DR images have been reported [22,23]. Integration of such techniques with simultaneous BV positioning might widen the VOIs available for 3D displacement evaluations.

The proposed VOI displacement estimation in conjunction with the $\mathrm{BV}$ positioning is expected to aid irradiation treatment decisions and to shift patient positioning from bony structure matching to tumor position matching by confirming that the VOI displacement is within the predetermined tolerance. Furthermore, CT volume data generated through DVM processing are expected to reflect the patient's anatomical condition in DR images involving the independent displacement of the VOI. Therefore, 3D-CT volumes can be obtained at the time of treatment with only two 2D-DR images, without any additional CT scans. Such CT volume data are expected to be applicable to dose distribution evaluations for patient positioning immediately prior to the irradiation treatment; this leads to more direct evaluations of the influence of the treatment in cases where the VOI displacements are properly reflected in the generated volumes, at least in and around the target and on the beam path. Further investigations should be conducted on the applicability of the 3D volumes generated by the DVM technique to dose distribution evaluations.

\section{Conclusions}

This paper presented the DVM technique and the evaluation of its performance in phantom and clinical patient cases. DRRs that reflected the patients' anatomical conditions could be obtained with DR images that showed displacements of VOIs from CT data, and 3D VOI displacements could be estimated independently and simultaneously with those of the BVs. The accuracies were shown to be comparable to those of the conventional 2D-3D matching technique. The proposed DVM technique is expected to be useful for independent displacement estimations of VOIs and BVs for patient positioning in radiation treatment. The DVM approach can also be extended to dose distribution evaluations from the generated CT volumes without additional CT scans, although further investigations would be required to assess the applicability of this technique for this purpose.

\section{Acknowledgements}

We would like to thank Ryosuke Okada, Satoshi Abe, and Saki Souda at Gunma University Hospital for their kind help with the image registrations. We would also like to acknowledge Takuma Nihira, Koichi Takanashi, and Masahiro Hasumi at Penguin System Co., Ltd. for their technical support. 


\section{Funding}

This work was partly supported by JSPS KAKENHI Grant Numbers JP25461904 and JP18K07744.

\section{Declaration of interest}

The authors have no conflict of interest to declare.

\section{References}

[1] Tashiro M, Ishii T, Koya J, Okada R, Kurosawa Y, Arai K, et al. Technical approach to individualized respiratory-gated carbon-ion therapy for mobile organs. Radiol Phys Technol 2013;6:356-66. https://doi.org/10.1007/s12194-013-0208-3.

[2] Irie D, Saitoh JI, Shirai K, Abe T, Kubota Y, Sakai M, et al. Verification of dose distribution in carbon ion radiation therapy for stage I lung cancer. Int $\mathrm{J}$ Radiat Oncol Biol Phys 2016;96:1117-23. https://doi.org/10.1016/j.ijrobp.2016.09.002.

[3] Houweling AC, Fukata K, Kubota Y, Shimada H, Rasch CR, Ohno T, et al. The impact of interfractional anatomical changes on the accumulated dose in carbon ion therapy of pancreatic cancer patients. Radiother Oncol 2016;119:319-25. https:// doi.org/10.1016/j.radonc.2016.03.004.

[4] Abe S, Kubota Y, Shibuya K, Koyama Y, Abe T, Ohno T, et al. Fiducial marker matching versus vertebral body matching: dosimetric impact of patient positioning in carbon ion radiotherapy for primary hepatic cancer. Phys Med 2017;33:114-20. https://doi.org/10.1016/j.ejmp.2016.12.018.

[5] Sakai M, Kubota Y, Saitoh JI, Irie D, Shirai K, Okada R, et al. Robustness of patient positioning for interfractional error in carbon ion radiotherapy for stage I lung cancer: Bone matching versus tumor matching. Radiother Oncol 2018;129:95-100. https://doi.org/10.1016/j.radonc.2017.10.003.

[6] Penny GP, Batchelor PG, Hill DL, Hawkes DJ, Weese J. Validation of a two- to threedimensional registration algorithm for aligning preoperative CT images and intraoperative fluoroscopy images. Med Phys 2001;28:1024-32. https://doi.org/10 $1118 / 1.1373400$.

[7] Fu D, Kuduvalli G. A fast, accurate, and automatic 2D-3D image registration for image-guided cranial radiosurgery. Med Phys 2008;35:2180-94. https://doi.org/ 10.1118/1.2903431.

[8] Markelj P, Tomaževič D, Likar B, Pernuš F. A review of 3D/2D registration methods for image-guided interventions. Med Image Anal 2012;16:642-61. https://doi.org/ 10.1016/j.media.2010.03.005.

[9] Kubota Y, Hayashi H, Abe S, Souda S, Okada R, Ishii T, et al. Evaluation of the accuracy and clinical practicality of a calculation system for patient positional displacement in carbon ion radiotherapy at five sites. J Appl Clin Med Phys 2018;19:144-53. https://doi.org/10.1002/acm2.12261.
[10] Ohno T, Kanai T, Yamada S, Yusa K, Tashiro M, Shimada H, et al. Carbon ion radiotherapy at the Gunma University Heavy Ion Medical Center: new facility setup. Cancers 2011;3:4046-60. https://doi.org/10.3390/cancers3044046.

[11] Ohno T. Particle radiotherapy with carbon ion beams. EPMA J 2013;4:9. https:// doi.org/10.1186/1878-5085-4-9.

[12] Maeda Y, Sato Y, Minami H, Yasukawa Y, Yamamoto K, Tamamura H, et al. Positioning accuracy and daily dose assessment for prostate cancer treatment using in-room CT image guidance at a proton therapy facility. Med Phys 2018;45:1832-43. https://doi.org/10.1002/mp.12858.

[13] Maeda Y, Sato Y, Shibata S, Bou S, Yamamoto K, Tamamura H, et al. Effects of organ motion on proton prostate treatments, as determined from analysis of daily CT imaging for patient positioning. Med Phys 2018;45:1844-56. https://doi.org/ 10.1002/mp.12869.

[14] Sun B, Yang D, Lam D, Zhang T, Dvergsten T, Bradley J, et al. Toward adaptive proton therapy guided with a mobile helical CT scanner. Radiother Oncol 2018;129:479-85. https://doi.org/10.1016/j.radonc.2018.08.021.

[15] Piotrowski T, Kaczmarek K, Bajon T, Ryczkowski A, Jodda A, Kaźmierska J. Evaluation of image-guidance strategies for prostate cancer. Technol Cancer Res Treat 2014;13:583-91. https://doi.org/10.7785/tcrtexpress.2013.600258.

[16] Adamczyk M, Piotrowski T, Adamiak E. Evaluation of combining bony anatomy and soft tissue position correction strategies for IMRT prostate cancer patients. Rep Pract Oncol Radiother 2012;17:104-9. https://doi.org/10.1016/j.rpor.2012.01. 005.

[17] Qin A, Gersten D, Liang J, Liu Q, Grill I, Guerrero T, et al. A clinical 3D/4D CBCTbased treatment dose monitoring system. J Appl Clin Med Phys 2018;19:166-76. https://doi.org/10.1002/acm2.12474.

[18] Combs SE, Ellerbrock M, Haberer T, Habermehl D, Hoess A, Jäkel O, et al. Heidelberg Ion Therapy Center (HIT): Initial clinical experience in the first 80 patients. Acta Oncol 2010;49:1132-40. https://doi.org/10.3109/0284186X.2010. 498432.

[19] Combs SE, Kessel KA, Herfarth K, Jensen A, Oertel S, Blattmann C, et al. Treatment of pediatric patients and young adults with particle therapy at the Heidelberg Ion Therapy Center (HIT): establishment of workflow and initial clinical data. Radiat Oncol 2012;7:170.

[20] Alaei P, Spezi E. Imaging dose from cone beam computed tomography in radiation therapy. Phys Med 2015;31:647-58. https://doi.org/10.1016/j.ejmp.2015.06.003.

[21] Murphy MJ, Balter J, Balter S, BenComo Jr JA, Das IJ, Jiang SB, et al. The management of imaging dose during image-guided radiotherapy: report of the AAPM Task Group 75. Med Phys 2007;34:4041-63. https://doi.org/10.1118/1.2775667.

[22] Terunuma T, Tokui A, Sakae T. Novel real-time tumor-contouring method using deep learning to prevent mistracking in X-ray fluoroscopy. Radiol Phys Technol 2018;11:43-53. https://doi.org/10.1007/s12194-017-0435-0.

[23] Zhang X, Homma N, Ichiji K, Abe M, Sugita N, Takai Y, et al. A kernel-based method for markerless tumor tracking in $\mathrm{kV}$ fluoroscopic images. Phys Med Biol 2014;59:4897-911. https://doi.org/10.1088/0031-9155/59/17/4897. 